\title{
Welwitschia mirabilis im Palmengarten
}

\author{
Veit M. Dörken \& Hilke Steinecke
}

\begin{abstract}
Welwitschia mirabilis is one of the most unique gymnosperms cultivated in the Palmengarten Frankfurt. In 2012 a spontaneous seedling was found in the Tropicarium. Morphology, biology and cultivation are described.

\section{Zusammenfassung}

Welwitschia mirabilis gehört zu den wohl eigentümlichsten Gymnospermen, die im Palmengarten Frankfurt kultiviert werden. 2012 wurde im Tropicarium erstmalig ein spontan entstandener Sämling im Bereich einer Mutterpflanze gefunden. Morphologie, Biologie und Kultur von Welwitschia werden erläutert.
\end{abstract}

\section{Einleitung}

Der Palmengarten Frankfurt beherbergt eine umfangreiche Sammlung von Nacktsamern, zu denen auch die aus dem Südwesten Afrikas stammende Gattung Welwitschia gehört, die nach ihrem Entdecker Friedrich Martin JoseF Welwitsch (1806-1872) benannt ist. Der österreichische Arzt und Botaniker sowie Direktor des Botanischen Gartens von Lissabon erforschte die junge portugiesische Kolonie Angola. Welwitsch war somit der erste Botaniker, der die Pflanzenwelt eines bestimmten afrikanischen Gebietes systematisch erkundete. Welwitschia mirabilis ist die einzige Art innerhalb der Welwitschiaceae, die mit Ephedraceae und Gnetaceae in der Ordnung Gnetales zusammengefasst werden (vgl. auch Kramer \& Green 1990, Chaw et al 2000). Es werden zwei Unterarten unterschieden: Die Subspecies mirabilis ist in Angola verbreitet, während die erst 2001 von LEUENBERGER neu beschriebene Subspecies namibiana, die auch das Staatswappen von Namibia ziert, in der Namibwüste Namibias heimisch ist.

\section{Welwitschia - eine ganz besondere Pflanze}

Im Palmengarten werden mehrere größere Exemplare im Nebelwüsten-Haus des Tropicariums und in der Botanischen Sammlung kultiviert. Das größte und älteste im Nebelwüsten-Haus vorhandene Exemplar ist eine Aussaat aus dem Jahr 1955. Es wurde vom damals noch zur Uni gehörenden Botanischen Garten Frankfurt erworben und 1989 in das gerade neu errichtete Tropicarium verpflanzt. Da hier ausreichend Platz vorhanden ist, wurde die Welwitschia nicht weiter in einer engen Tonröhre kultiviert, in der sie zuvor über Jahrzehnte gehalten worden war. Im Nebelwüsten-Haus wird nicht genebelt; stattdessen werden einzelne Pflanzenindividuen, so auch die Welwitschien, gezielt gegossen. Sie bekommen auf diese Art und Weise mehr Wasser als in der Namibwüste. Sie wachsen im Palmengarten recht schnell. Die Blätter können hier einen jährlichen Zuwachs von bis 30 Zentimeter pro Jahr aufweisen, selbst über den Winter legen sie $1-3 \mathrm{~cm}$ zu. Die älteste Welwitschia hat aktuell rund $3 \mathrm{~m}$ lange grüne Blattabschnitte, dazu kommen die ebenso langen abgestorbenen und zerfransten braunen Blattenden. Welwitschien, die 1996 ausgesät wurden, haben immerhin bereits 1-1,5 m lange Blätter.

Mit einem maximalen Alter von rund 2000 Jahren ist Welwitschia sehr langlebig. Da der Vegetationspunkt sein Längenwachstum recht früh einstellt, erreichen selbst alte Exemplare nur eine Höhe von $1 \mathrm{~m}$. Das Hypokotyl, also der Bereich zwischen Wurzelhals und den zwei Keimblättern, ist kräftig entwickelt und lang gestreckt. Das darauf folgende Epikotyl, der Bereich zwischen Keim- und Folgeblättern, ist nur schwach ausgeprägt und stark gestaucht. Der Stamm wird daher fast ausschließlich vom Hypokotyl gebildet. Im Alter ist der Stamm rübenartig und im oberen Bereich breit-tellerförmig abgeflacht.

Welwitschia entwickelt neben einem hohen Anteil an feinen Faserwurzeln im oberen Boden- 


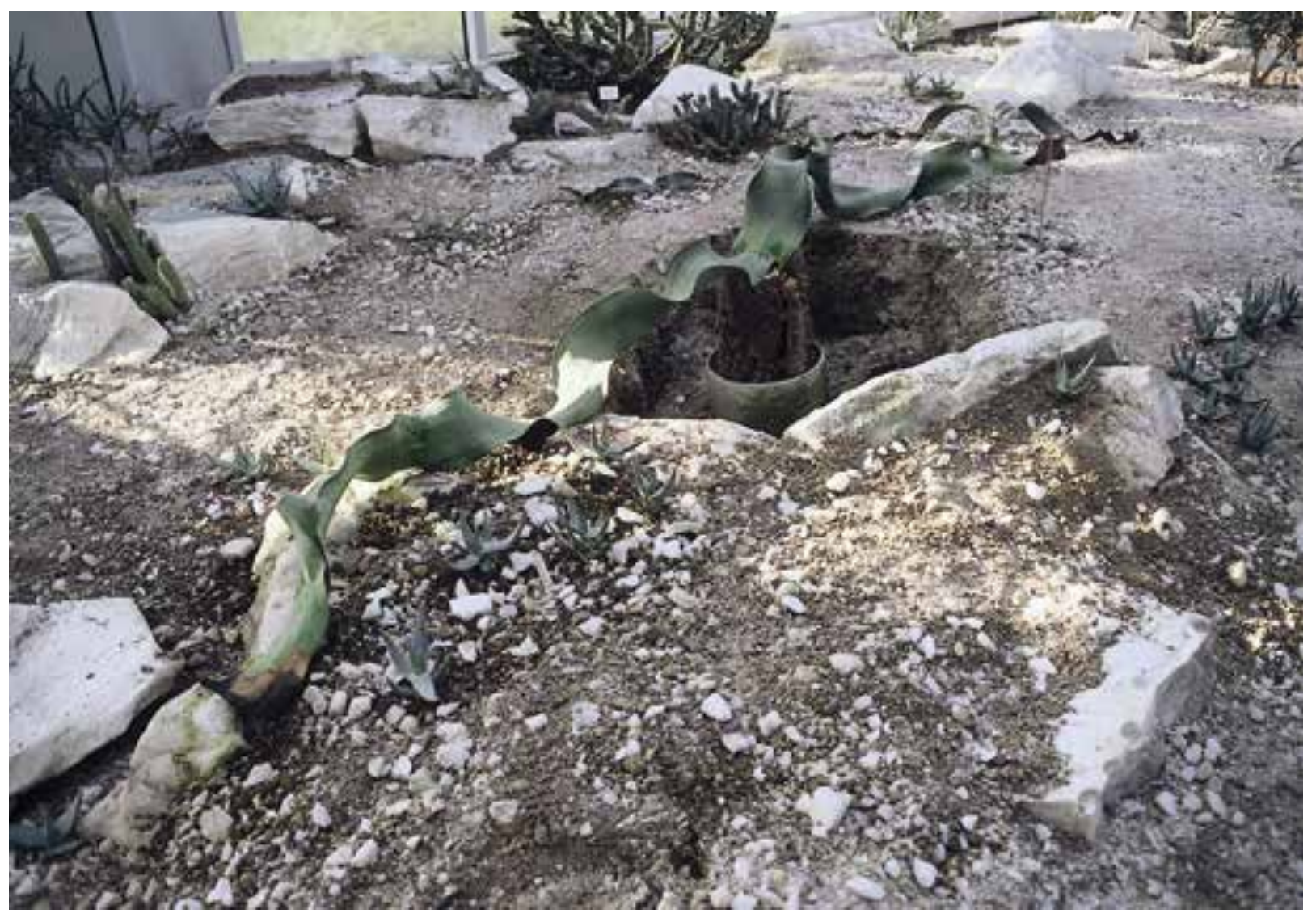

horizont, auf die man noch in einem Radius von mehreren Metern um die Pflanze treffen kann, eine lange, in die Tiefe reichende, rübenartige Wurzel mit Speicherfunktion (KuTsCherAMitter \& Lichtenberger 1997). Sie reagiert sehr empfindlich gegenüber Eingriffen, sodass bei kultivierten Welwitschien frühzeitig der endgültige Standort ausgewählt werden sollte, da ein späteres Verpflanzen schwierig ist und nur sehr schlecht vertragen wird.

Die Belaubung von Welwitschia ist einzigartig. Unmittelbar auf die Keimblätter werden zwei Folgeblätter ausgebildet, Primärblätter fehlen. Keim- und Folgeblätter sind die einzigen photosynthetisch aktiven Blätter, die zeitlebens hervorgebracht werden. Auf die Folgeblätter entwickeln sich noch zwei kleine Schuppenblätter. Die beiden bandförmigen grünen Blätter weisen eine für Gymnospermen

Abb. 1: Die älteste Welwitschia mirabilis des Palmengartens ist neu in der Nebelwüste gepflanzt (2. Februar 1989). typische Parallelnervatur auf. Sie sterben an der Spitze ab, sodass selten Blattlängen über $4 \mathrm{~m}$ auftreten. Am Blattgrund wird kontinuierlich neues Blattgewebe gebildet. Mit zunehmendem Umfang des Stammes reißen die Blattbasen und vor allem auch das Bildungsgewebe ein, so dass ältere Individuen scheinbar mehrere Blätter haben. Auf den Blättern selbst sind Jahreszuwächse anhand deutlich hellerer Bereiche, die im Winter gebildet werden, erkennbar.

\section{Fortpflanzungsorgane}

Welwitschia ist zweihäusig, d. h. es gibt männliche und weibliche Individuen. Männliche sowie weibliche Blüten stehen in aufrechten zapfenartigen Strukturen, die sich aus zahlreichen Einzelblüten zusammensetzen. Welwitschien sind hauptsächlich insektenbestäubt (Wespen und Wanzen, vgl. Scherer 2006).

Die scheinbare Einzelblüte in einem männlichen Zapfen ist ein verzweigtes System und entspricht damit einem Teilblütenstand. Jeder dieser Teilblütenstände umfasst drei Einzelblüten. Seitlich stehen zwei männliche Blüten, dazwi- 

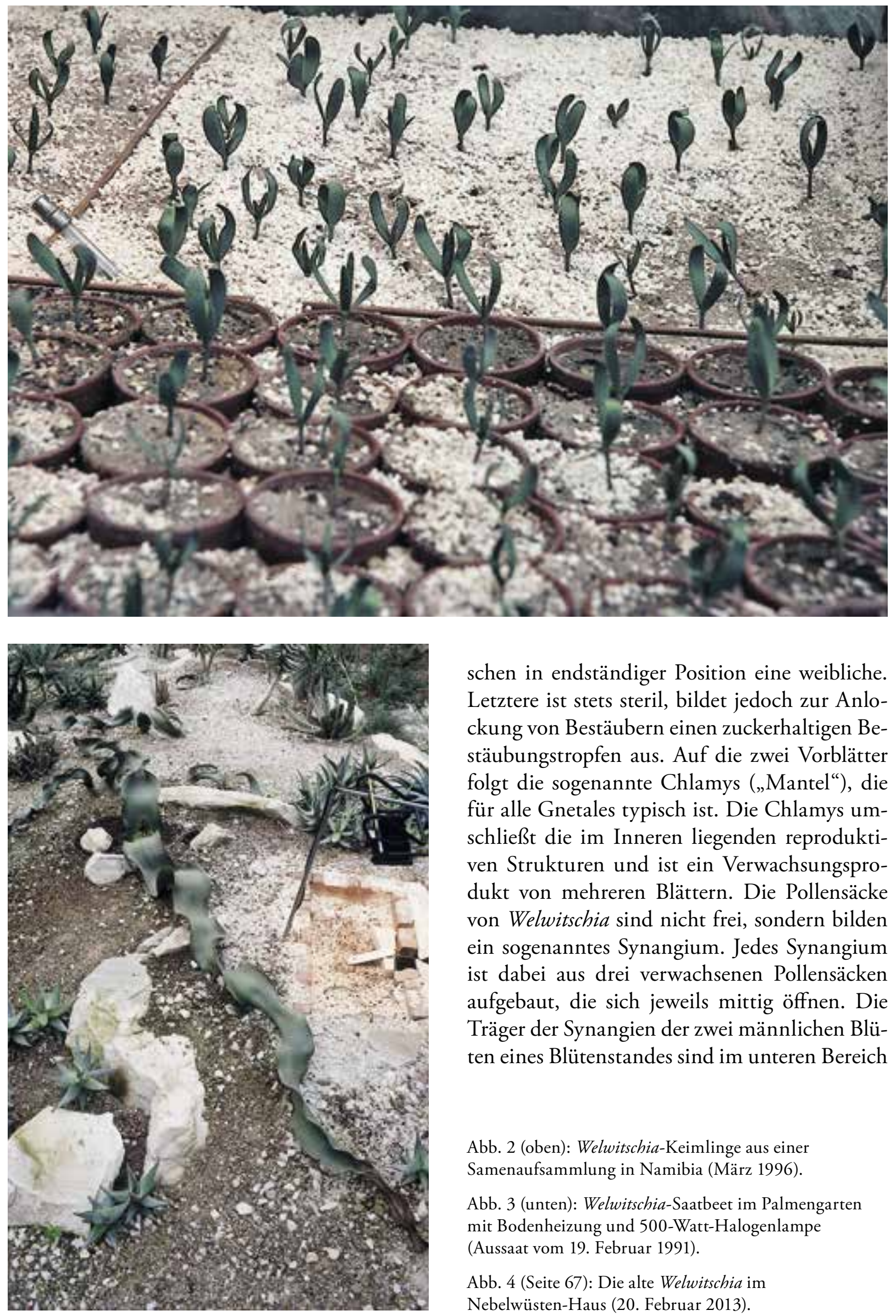

schen in endständiger Position eine weibliche. Letztere ist stets steril, bildet jedoch zur Anlockung von Bestäubern einen zuckerhaltigen Bestäubungstropfen aus. Auf die zwei Vorblätter folgt die sogenannte Chlamys („Mantel“), die für alle Gnetales typisch ist. Die Chlamys umschließt die im Inneren liegenden reproduktiven Strukturen und ist ein Verwachsungsprodukt von mehreren Blättern. Die Pollensäcke von Welwitschia sind nicht frei, sondern bilden ein sogenanntes Synangium. Jedes Synangium ist dabei aus drei verwachsenen Pollensäcken aufgebaut, die sich jeweils mittig öffnen. Die Träger der Synangien der zwei männlichen Blüten eines Blütenstandes sind im unteren Bereich

Abb. 2 (oben): Welwitschia-Keimlinge aus einer Samenaufsammlung in Namibia (März 1996).

Abb. 3 (unten): Welwitschia-Saatbeet im Palmengarten mit Bodenheizung und 500-Watt-Halogenlampe (Aussaat vom 19. Februar 1991).

Abb. 4 (Seite 67): Die alte Welwitschia im

Nebelwüsten-Haus (20. Februar 2013). 


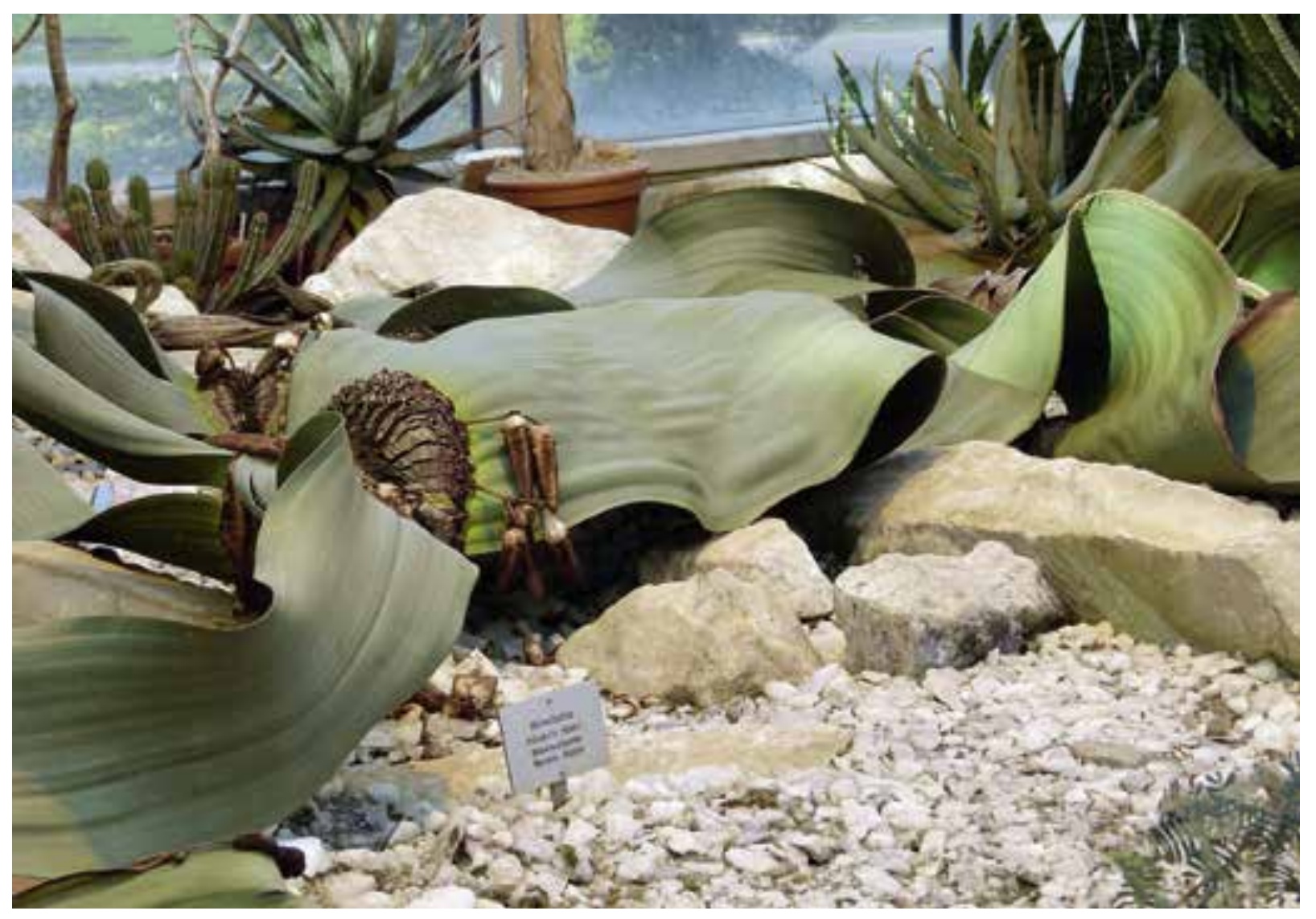

zu einer gemeinsamen ringförmigen Struktur verwachsen (vgl. auch Sykes i9i I, MundRY \& STÜTZEL 2004).

Die beiden Unterarten lassen sich aufgrund von Struktur und Färbung ihrer männlichen Zapfen deutlich voneinander unterscheiden. Die purpur-braunen Zapfen der Subspecies mirabilis weisen einen vergleichsweise kurzen Zapfenstiel auf. Die einzelnen Zapfen sind relativ lang, die Tragblätter der einzelnen männlichen Blüten überlappen sich stark. Bei der Subspecies namibiana stehen die bläulich-grünen bis lachsfarbenen wesentlich kürzeren Zapfen an langen Stielen. Die Tragblätter der einzelnen männlichen Blüten überlappen sich hier nicht oder nur kaum. Zudem erfolgt die Blütezeit rund drei Wochen später als bei der Unterart mirabilis (LEUENBERGER 2001).

Die weiblichen Blüten stehen ebenfalls in aufrechten Zapfen. Jede Einzelblüte steht in der Achsel eines Tragblattes und umfasst nur eine Samenanlage. Auch hier ist eine Chlamys vorhanden. Diese differenziert sich mit zunehmender Samenreife in eine äußere pergamentartige
Schicht, die den Samenflügel ausbildet und der späteren Windausbreitung dient, während die inneren Schichten der Chlamys stark verholzen und so dem Schutz des Embryos dienen (vgl. Mundry \& Stützel 2004). Aufgrund von Gewebeschrumpfung zerfallen die weiblichen Zapfen zum Zeitpunkt der Samenreife. Die geflügelten Samen werden freigesetzt und durch Wind ausgebreitet.

\section{Blühende Welwitschien und Sämlinge im Palmengarten}

Die größeren Exemplare bringen regelmäßig Pollen- bzw. Samenzapfen hervor, wobei die älteste Palmengarten-Welwitschia ein weibliches Exemplar ist. Sie wurde bereits mehrfach erfolgreich mit Pollen eines anderen Exemplars aus dem Palmengarten bestäubt, sodass sich keimfähige Samen entwickelten. Die Samen keimen relativ gut nach etwa einer Woche und behalten ihre Keimfähigkeit, zumindest bei tiefgefrorenen Samen, lange bei.

Im Botanischen Garten Frankfurt wurde aufgrund des Ausfalls einer Tiefkühltruhe ein 

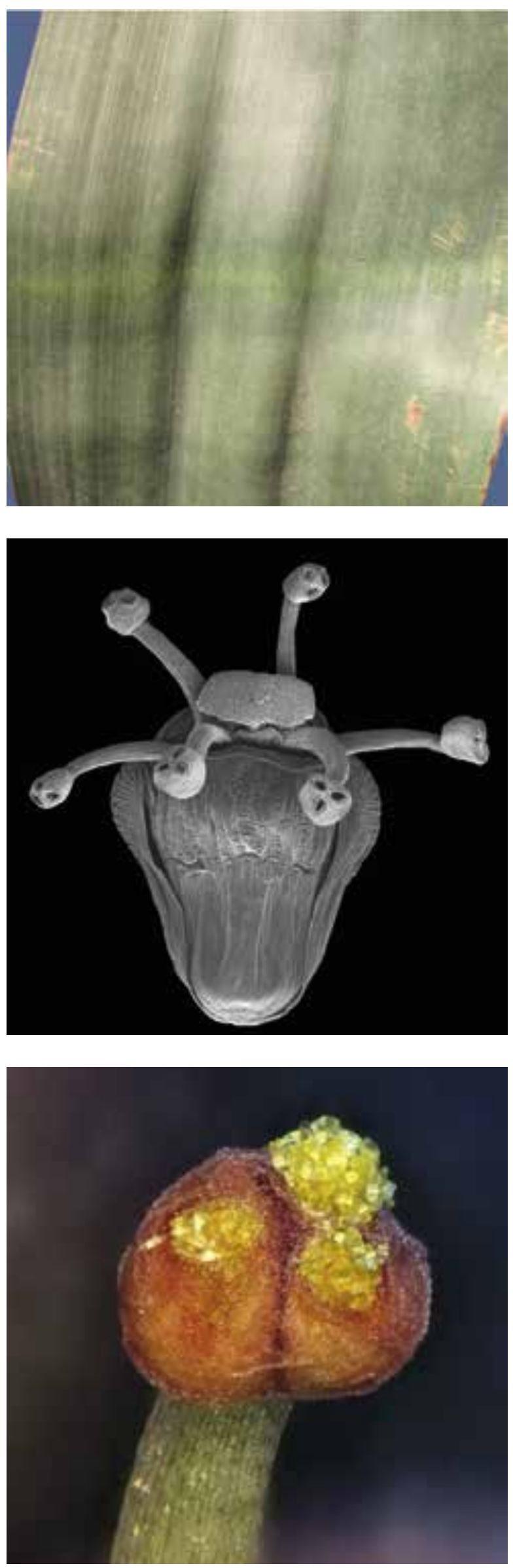

Keimungstest durchgeführt. Ein etwa 40 Jahre lang bei $-18^{\circ} \mathrm{C}$ eingefrorener Welwitschia-Same (Samenbank des Botanischen Gartens) der aus Angola stammenden, selteneren Unterart wurde 2011 ausgesät und keimte sofort. Erst in der Keimphase sind Welwitschia-Sämlinge durch Pilzbefall stark gefährdet, sodass ohne die Verwendung von Fungiziden mit Ausfällen zu rechnen ist. Auch der aus dem „Dornröschenschlaf aufgeweckte" Keimling hatte leider keine lange Lebensdauer.

Im Jahr 2012 hat sich im Palmengarten neben der alten Welwitschia, die manuell bestäubt wurde und daraufhin fertile Samen bildete, spontan ein Sämling angesiedelt, ein wahrscheinlich bislang einzigartiges Phänomen in der Gewächshauskultur. Fungizide zum Schutz des Keimlings wurden nicht eingesetzt. Das Keimblattstadium hat der Sämling bereits hinter sich, die beiden Laubblätter haben nach etwa einem Jahr eine Länge von rund $8 \mathrm{~cm}$ und eine Breite von etwa $0,4 \mathrm{~cm}$.

\section{Tipps zur Aussaat}

Für die Anzucht von Welwitschien an der Universität Konstanz hat sich eine Aussaat in einer speziellen Klimakammer, in der Temperatur, Belichtung und Luftfeuchte kontrolliert werden können, bewährt. Aussaatversuche in einem Lichtthermostat bei einem simulierten 12-Stunden-Tag mit konstanter Temperatur $\left(30^{\circ} \mathrm{C}\right)$ sowie Belichtung $\left(100 \mathrm{~m} \mu \mathrm{mol} / \mathrm{m}^{2} / \mathrm{s}\right)$ bei ausreichender Luftzirkulation haben gezeigt, dass von 30 Samen innerhalb von vier Tagen 28 keimten. Als Aussaatsubtrat eignet sich ein zuvor sterilisiertes Torf-Sand-BimsGemisch (2:1:1). Da das Wurzelsystem von Welwitschia sehr empfindlich auf Eingriffe jeglicher Art reagiert, wurden die Samen einzeln in langen röhrenförmigen Gefäßen ausgesät. Die Samen sollten bei der Aussaat in Samen-

Abb. 5 (oben): Jahresgrenze auf dem Blatt.

Abb. 6 (Mitte): Rasterelektronische Aufnahme eines männlichen Blütenstandes. Gut erkennbar sind die Synangien.

Abb. 7 (unten): Synangium von Welwitschia mirabilis. 
kornstärke mit Substrat vollständig bedeckt werden. Vor der Aussaat müssen die papierartigen Samenflügel entfernt werden, da diese recht schnell von Schimmelpilzen befallen werden. Um einen Pilzbefall generell zu vermeiden, hat sich die Behandlung der Samen vor der Aussaat mit einem Fungizid bewährt. Um die Gefährdung durch später auftretende Pilzerkrankungen (z. B. Umfallkrankheit) zu minimieren, sollte auch nach der Keimung in regelmäßigen Abständen dem angewärmten Gießwasser ein Fungizid beigemischt werden. Ab dem Zeitpunkt der Keimung darf das Substrat auf keinen Fall mehr austrocknen, da die Sämlinge empfindlich auf Trockenheit reagieren.

Für die Weiterkultur ist es nicht zwangsweise nötig, die Welwitschien in hohen Tonröhren zu halten, wie es früher in vielen Sammlungen praktiziert wurde. Im Palmengarten beispielsweise wurden Welwitschien in normalen Blumentöpfen ausgesät. Sobald die Wurzel aus dem Loch des Blumentopfes herauswächst, muss sie gekappt werden. Sie länger durchtreiben zu lassen und erst später einzukürzen, verträgt Welwitschia nicht. Wenn bisher im Topf kultivierte Welwitschien im Gewächshausbeet ausgepflanzt werden sollen, muss das Pflanzloch gut vorbereitet sein. Die Pflanzen werden nicht aus dem Topf herausgezogen. Vielmehr wird der Tontopf vorsichtig über dem Pflanzloch zerschlagen, die Scherben entfernt und die Welwitschia ganz behutsam an ihren neuen Wuchsort gesetzt.

\section{Dank}

Für kritische Durchsicht des Manuskriptes und Hinweise zur Welwitschia-Kultur danken wir Kristina BaKhtiari-Kish, Roland Rudolph und Andreas König und für die Durchführung der Aussaatversuche Ulrike Sick (Botanischer Garten Konstanz).

Abb. 8 (oben): Weibliches Individuum einer Welwitschia mirabilis mit Zapfen.

Abb. 9 (Mitte): Welwitschia mirabilis mit Bestäubunsgstropfen.

Abb. 10 (unten): Welwitschia-Keimling.
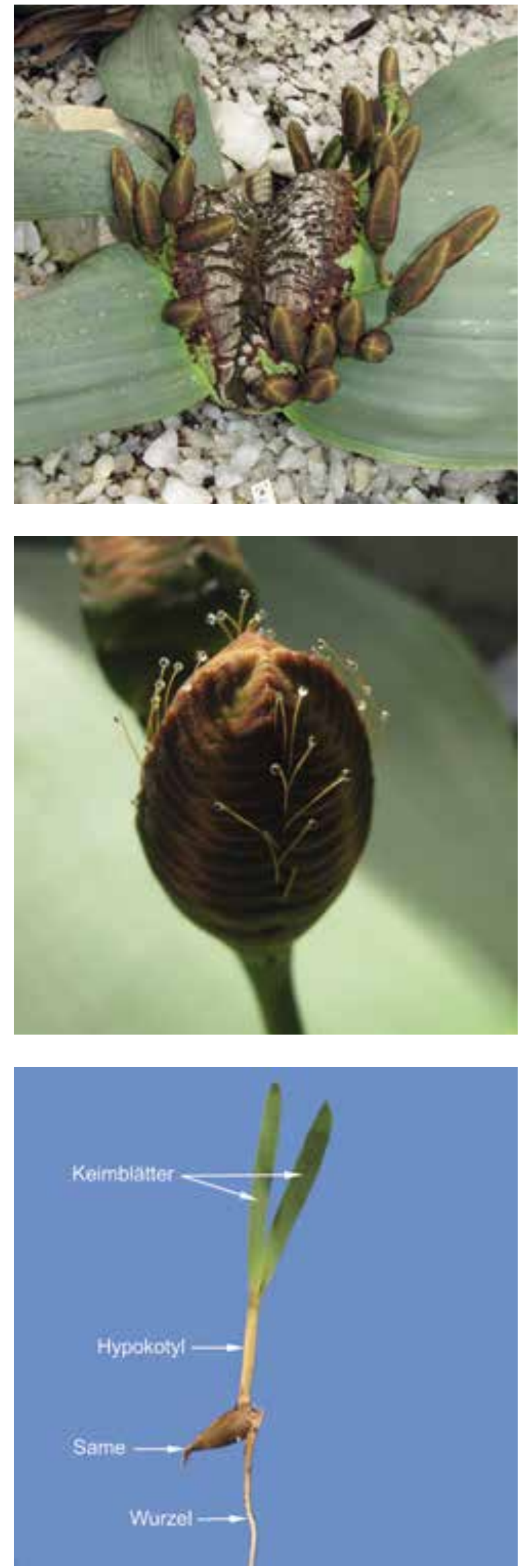


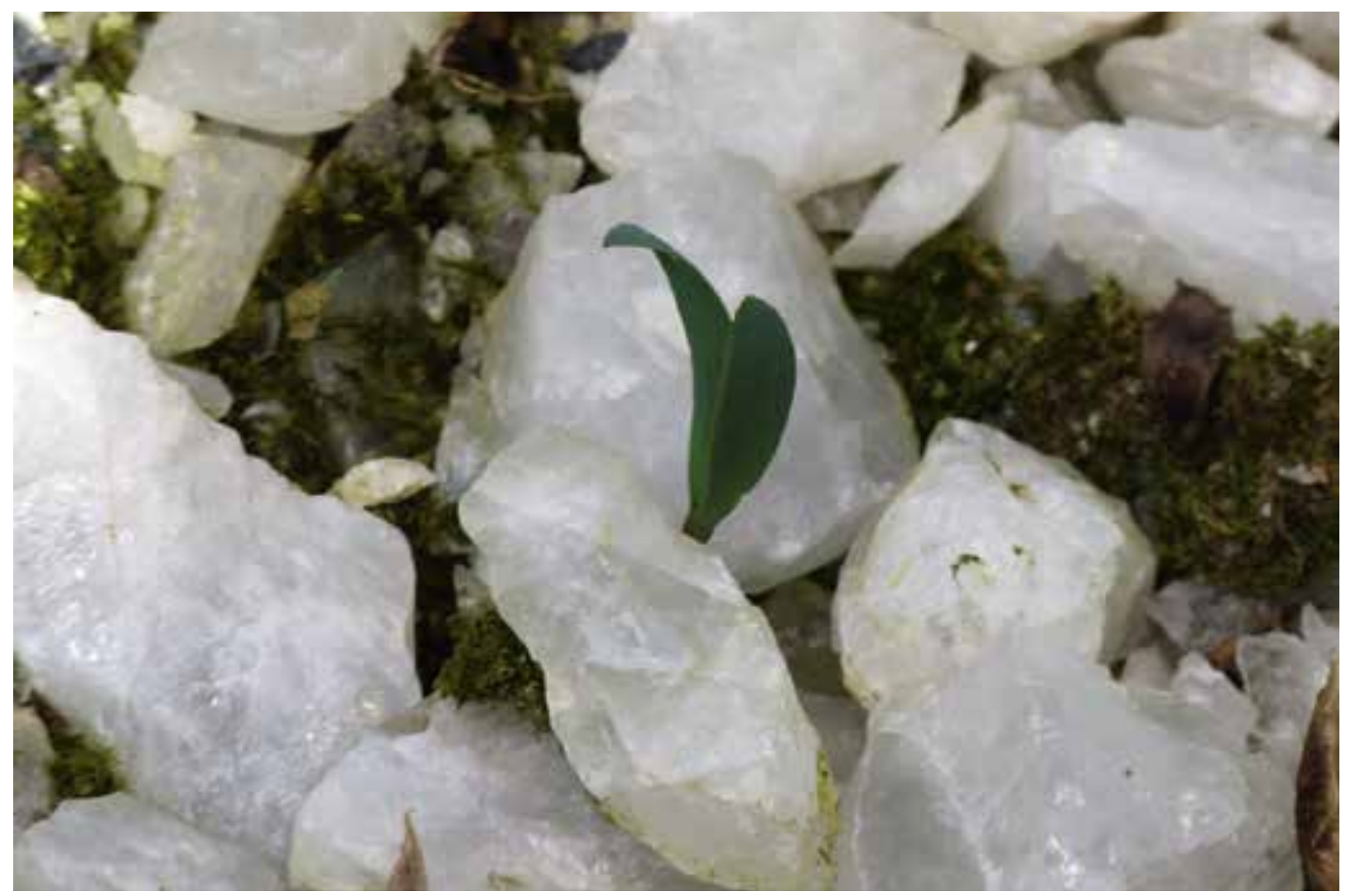

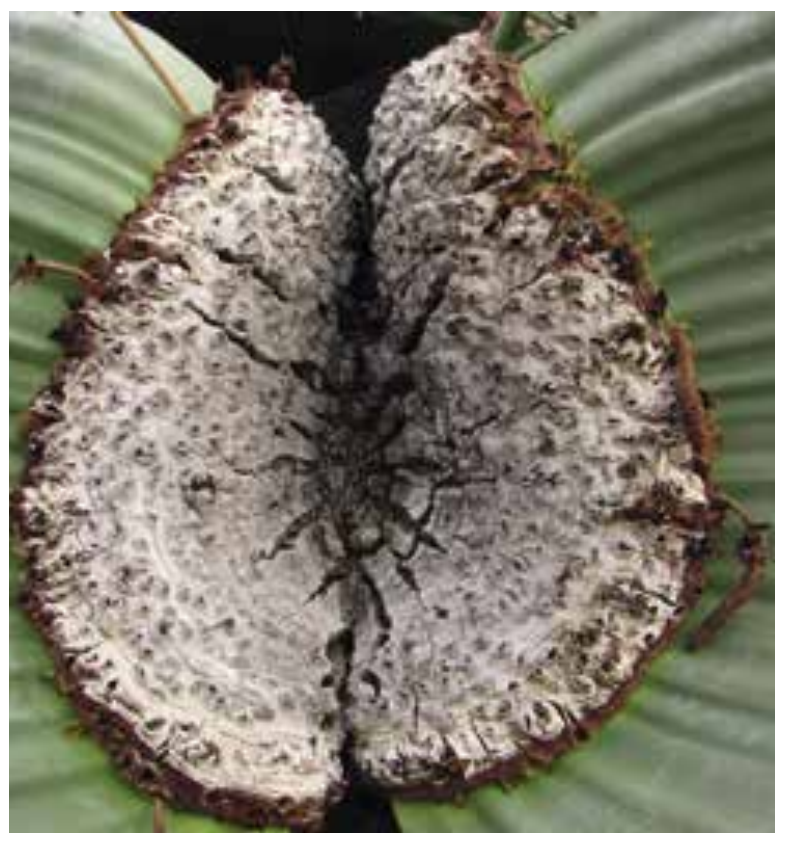

\section{Literatur}

Chaw, S. M., Parkinson, C. L., Cheng, Y., Vincent, T. M. \& Palmer, J. D. 2000: Seed plant phylogeny inferred from all three plant genomes: Monophyly of extant gymnosperms and origin of Gnetales from conifers. - PNAS, 97: 4086-4091.

Kramer, K. U. \& Green, P. S. 1990: The families and genera of vascular plants, Pteridophytes and Gymnosperms, edited by K. Kubitzki. - Heidelberg.
Kutschera-Mitter, L. \& Lichtenberger, E. 1997: Die Wurzel - Hilfe für das Überleben von Welwitschia mirabilis und anderer Arten der Namib. - Palmengarten 61: 31-40. Leuenberger, B. E. 2001: Welwitschia mirabilis (Welwitschiaceae), male cone characters and a new subspecies. - Willdenowia 31: 357-381.

Mundry, M. \& STützel, T. 2004: Morphogenesis of the reproductive shoots of Welwitschia mirabilis and Ephedra distachya (Gnetales), and its evolutionary implications. - Organisms, Diversity \& Evolution 4: 91-108. Scherer, R. 2006: Welwitschia mirabilis und ihre Begleitarten bei Swakopmund. - Palmengarten 70: 34-38. Sykes, M. G. 1911: The anatomy and morphology of the leaves and inflorescences of Welwitschia mirabilis. - Phil. Trans. Roy. Soc., series B, 201: 179-226.

\section{Internetseite}

http://www.mobot.org/MOBOT/research/APweb/. Stevens, P. F. 2001: Angiosperm phylogeny website. Version 9, June 2009. http://www.mobot.org/mobot/ research/apweb/

Abb. 11 (oben): Welwitschia-Keimling im NebelwüstenHaus des Palmengartens.

Abb. 12 (unten): Spross-Scheitel einer älteren Welwitschia. 